\title{
ترقية نتيجة تعلم النحو باستخدام طريقة دورة الألعاب الجماعية في المدرسة المنزلية شعائر الله بيكاسي
}

\section{Muhammad Kamal bin Abdul Hakim}

Universitas Negeri Jakarta

m.kamal.ah@unj.ac.id

\section{Romdani}

Universitas Negeri Jakarta

romdani.unj@unj.ac.id

\section{Dian Priandini}

UIN Maulana Malik Ibrahim Malang

dianpriandini1@gmail.com

DOI: http://dx.doi.org/10.18326/lisania.v3i2.180-197

\begin{abstract}
The purpose of this research was to know the enhancement of learning results of Arabic grammar by using the Teams Games Tournament method in 10th grade at Home Schooling Sya'airullah Bekasi. The method used was the classroom action research with the study the student of Home Schooling Sya'airullah Bekasi 10th grade which amounted to 40 students as the sample. The research was conducted at Home Schooling of Sya'airullah Bekasi in March - April 2019. The classrom action research was conduct by using the Kemmis and Mc Taggart cycle model through four stages: planning, action, observation and reflection. The instruments used were learning results tests and observation sheets. Learning results tests were used to measure the success of students in understanding arabic grammar, while the observation sheet was used to measure teacher and student performance in learning. The result obtained by the students during the research was average 70.87 in the first cycle and 88.52 in the second one. Then, the enhancement from the first cycle to the second cycle was 17.65. Based on the results above, the researcher concluded that the Teams Games Tournament method can improve students' ability to understand the science arabic grammar and can improve the learning results of a rabic grammar in 10th grade students at Home Schooling Sya'airullah Bekasi.
\end{abstract}

Keywords: Learning Results Arabic Gramma, Teams Games Tournament Method, Classroom Action Research 


\begin{abstract}
Abstrak
Penelitian ini bertujuan untuk mengetahui peningkatan hasil belajar nahwu dengan menggunakan metode Teams Games Tournament pada siswa kelas 10 Home Schooling Sya'airullah Bekasi. Metode penelitian yang digunakan dalam penelitian ini adalah penelitian tindakan kelas (PTK). Sumber data dalam penelitian ini adalah siswa kelas 10 Home Schooling Sya'airullah dengan jumlah 40 siswa. Penelitian dilaksanakan di Home Schooling Sya'airullah Bekasi pada bulan Maret - April 2019. Penelitian tindakan kelas ini mengacu pada model Kemmis dan Mc Taggart. Berikut tahapan penelitian ini adalah perencanaan, pelaksanaan, observasi dan refleksi. Penelitian ini dilaksanakan dalam dua siklus. Instrumen yang digunakan adalah tes hasil belajar dan lembar observasi. Tes hasil belajar digunakan untuk mengukur keberhasilan siswa dalam memahami ilmu nahwu. Lembar observasi digunakan untuk mengukur kinerja guru dan siswa dalam pembelajaran. Nilai yang dicapai oleh siswa selama penelitian berlangsung yaitu nilai rata-rata siklus I adalah 70,87 dan nilai rata-rata siklus II adalah 88,52. Dan peningkatan dari siklus I ke siklus II yaitu 17,65. Berdasarkan hasil penelitian, peneliti menyimpulkan bahwa metode Teams Games Tournament dapat meningkatkan kemampuan siswa dalam memahami ilmu nahwu dan dapat meningkatkan hasil belajar nahwu pada siswa kelas 10 di Home Schooling Sya'airullah Bekasi.
\end{abstract}

Kata Kunci: Hasil Belajar, Nahwu, Metode Teams Games Tournament, PTK

$$
\begin{aligned}
& \text { إن أحد أدوار المعلم في عملية التعليم والتعلم هو المخطط، حيث يجب على المعلم } \\
& \text { أن يضع خطط التعليم المناسبة بحالة الطلاب، سواء كان في استخدام النموذج أو طرائق } \\
& \text { التعليم أو أساليبه، وذلك لتحقيق أهداف عملية التعليم التعلم. باختيار طرائق التعليم } \\
& \text { وأساليبه المناسبة، يمكن للمعلم أن يزيد نشاط الطلاب في التعلم، ونشاط تعلم الطلاب } \\
& \text { هو من إحدى العناصر الأساسية المهمة لنجاح عملية التعليم (Wibowo, 2016). ويحدث } \\
& \text { نشاط الطالب في عملية التعليم عند تحقيق الأشياء التالية: التعليم يتركز على الطلاب، } \\
& \text { دور المعلم كالمرشد حتى تحدث خبرات التعلم أكثر تحقيقا لمدف عملية التعليم والتعلم وتركز } \\
& \text { إدارة أقصى لكفاءة عملية التعليم بإبداع الطلاب وإتقان المفاهيم والقياس المستمر في } \\
& \text { مختلف جوانب المعارف، والمواقف، والمهارات (Priansa, 2017:41). من هذا الرأي نعرف } \\
& \text { أن التعليم الذي يتركز على الطلاب هو الذي يدفع نشاط الطلاب في التعلم. }
\end{aligned}
$$


التعليم الذي يتركز على الطلاب هو التعليم الذي يركز على نشاط الطلاب في تنفيذ عملية التعليم والتعلم. في إحدى الدراسات، كان إدراك الطلاب لبيئة تعليمية إيجابية

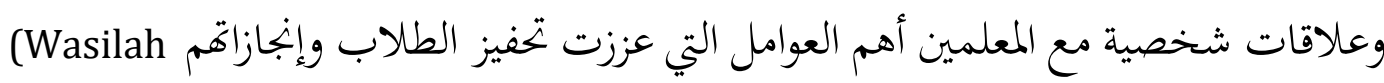

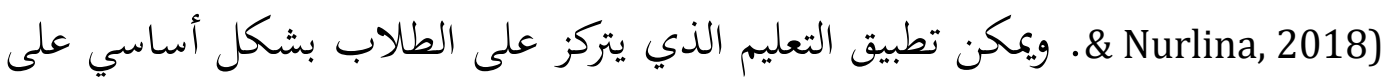

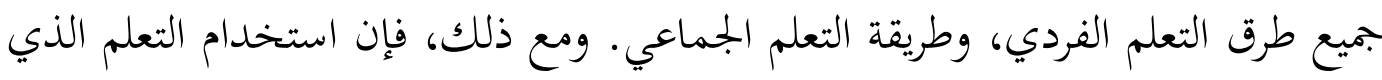

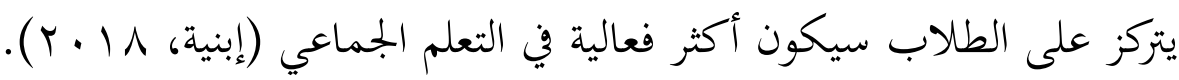

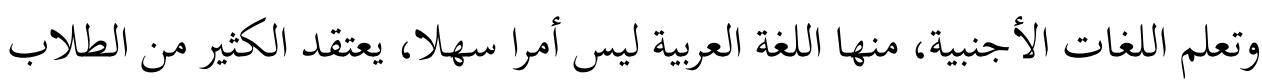

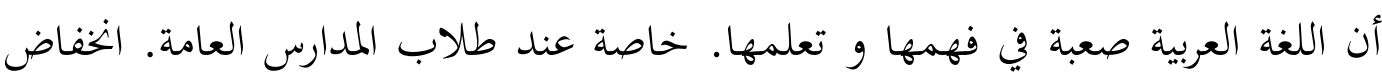
اهتمام الطلاب بتعلم اللغة العربية يرجع إلى استخدام طرق التعليم غير مبتكرة و عملية التعلم يتركز على المعلم فقط، مما يجعل الطلاب غير ناشطين في التعلم. واستخدام طريقة التعلم المبتكرة والممتعة يمكن أن يزيد انتباه الطلاب وتركيزهم بالتعلم و يساعدهم في فهم

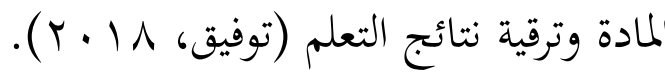
طريقة تعليم اللغة العربية في المدرسة المنزلية (Home Schooling) شعائر الله بيكاسي جوى الغربية هي الطريقة التعلمية التقليدية، مثل طريقة المحاضرة التي تتركز على المعلم فقط

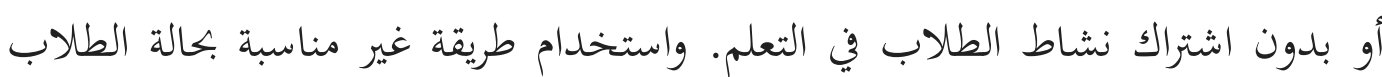
يسبب إلى عدم تطور نشاط الطلاب. والمشكلة الأخرى في المدرسة المنزلية هي كثير من الطلاب يحتسبون أن اللّغة العربيّة صعبة بخاصة علم النحو، لأن كثيراً من الطلاب لم يدرسوا اللغة العربية من قبل. ومن هذه المشاكل العديدة الموجودة، يرغب الباحث في استخدام

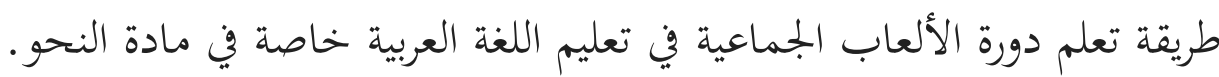

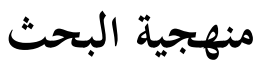

أجري هذا البحث الإجرائي في المدرســة المنزلية شـعائر الله بيكاسـي للصـف

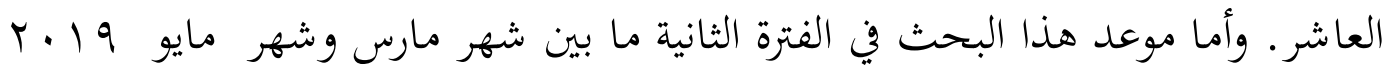
م. وطريقة البحث المستخدمة هي بحث الإجراء الصفي باستخدام طريقة دورة الألعاب

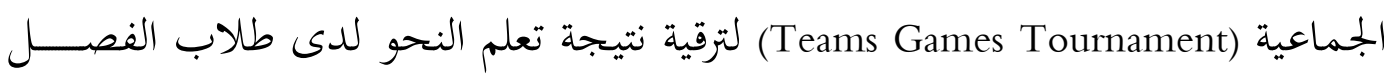


العاشر في المدرسة المنزلية شعائر الله بيكاسي. والنمودج المستخدم ما قدمه كميس وميك تيغارت (Kemmis and Mc Taggart): التخطيط، والعمل، والملاحظة، والانعكاس.

ويمكن تصـور خطوات البحث الإجرائي الصـفي بما يلي: :Anugrah, 2019)

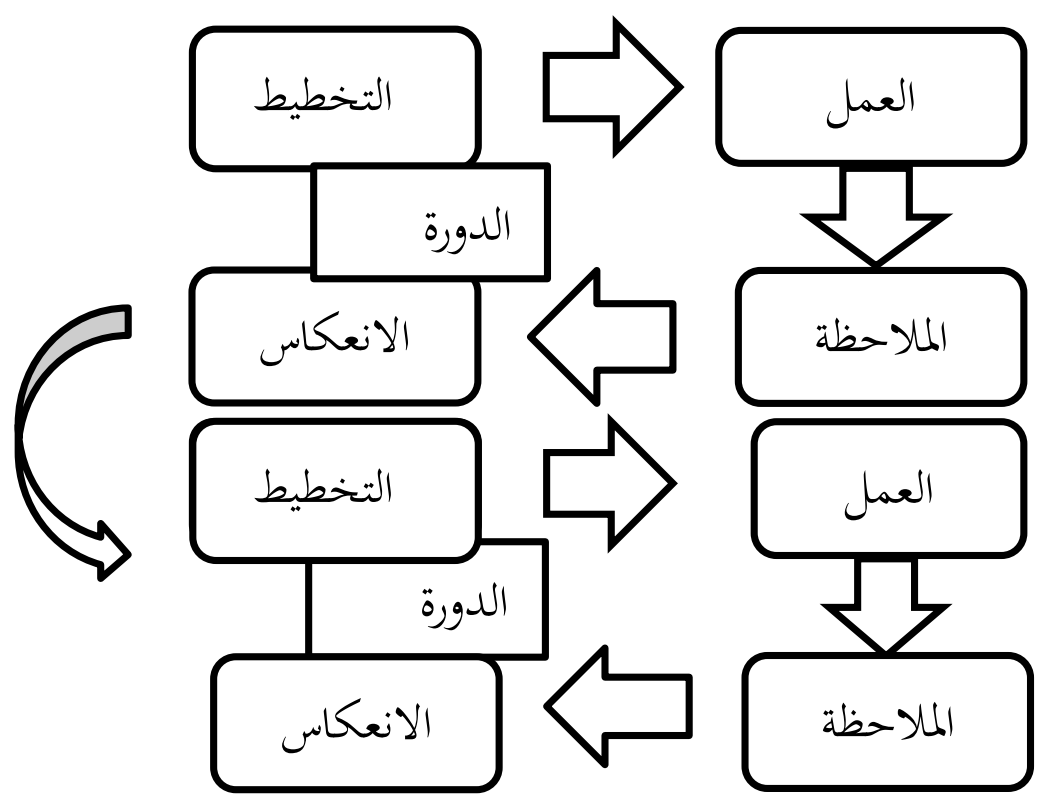

الرسم 1 ـ خطوات بحث الإجراء الصفي عند كميس وميك تيغارت

بدأ البحث الإجرائى الصـفي في الدورة الأولى التي تتكون من أربع نشـطات.

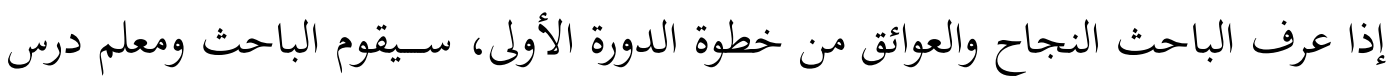

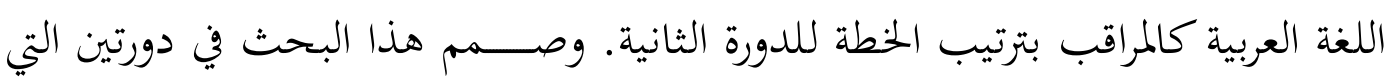

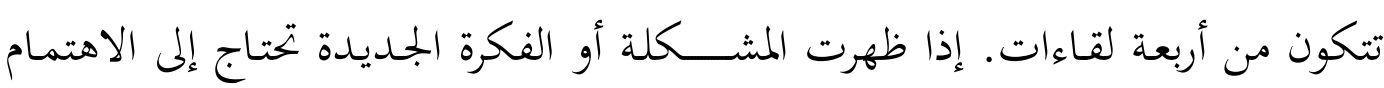

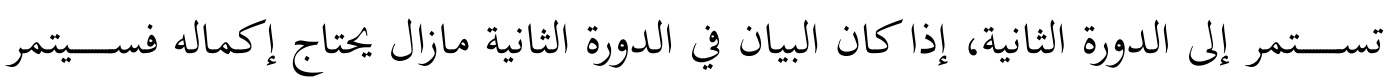

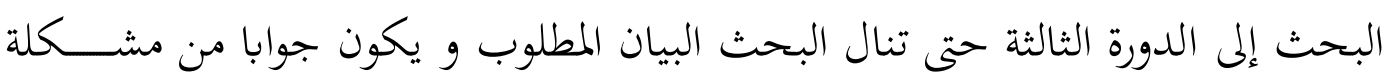
البحث.

$$
\begin{aligned}
\text { الأنشطة قبل البحث: هو تصميم التدخل العملي في بحث الإجراء الصفي } & \text { الأنشطة قبل البحث. }
\end{aligned}
$$




\section{أ. الاتصال بمدير المدرسة للحصول على الإذن للبحث. ب. الملاحظة في عملية الدراسة في الفصل. ج. تحليل المشكلة. إجراءات البحث في الدورة الأولى بالخطوات الآتية: مرحلة تخطيط العمل} أ. تخطيط خطة التعليم باستخدام طريقة دورة الألعاب الجماعية. ب. تصميم خطة التعليم. ج. إعداد ورقة المراقبة للأنشطة التعليمية للمعلم كالمراقب. د. إعداد ورقة المراقبة للأنشطة التعليمية للطلاب. هـ إعداد وثائق أنشطة التعلم.

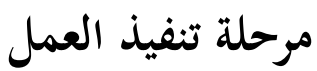

أ. إعداد الطلاب في مشاركة عملية التعليم و التعلم. ب.إجراء التعليم باستخدام طريقة دورة الألعاب الجماعية.

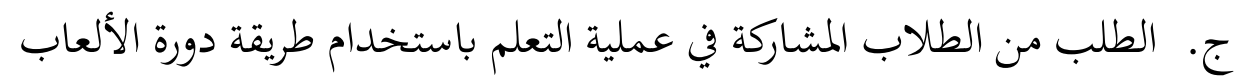
الجماعية.

د. تسجيل الأحداث و الأشياء المهمة التي تحدث أثناء الدراسة. مرحلة الملاحظة

أ. مراقبة وتسجيل عملية التعلم التي تحدث أثناء الدورة الأولى، وهذه الملاحظة قد أجرى الباحث بمساعدة المراقب. ب. توثيق أنشطة التعليم و أنشطة الطلاب في الفصل الدراسي. مرحلة الانعكاس

أ. يناقش الباحث نتيجة الملاحظة مع المراقب للحصول على نتيجة الملاحظة أو الانعكاس لمعرفة عيوبها و تقديم الاقتراحات من تلك الدورة. ب. تخطيط العمل للدورة الثانبة، تعتمد على نتيجة الانعكاس في الدورة الأولى. 
إجراءات هذا البحث في الدورة الثانية بالخطوات الآتية: مرحلة تخطيط العمل

أ. تخطيط خطة التعليم باستخدام طريقة دورة الألعاب الجماعية. ب. تصميم خطة التعليم. ج. إعداد ورقة المراقبة للأنشطة التعليمية للمعلم. د. إعداد ورقة المراقبة للأنشطة التعليمية للطلاب. هـ. إعداد وثائق أنشطة التعلم. مرحلة تنفيذ العمل أ. إعداد مشاركة الطلاب في عملية التعليم و التعلم. ب.اجراء التعليم باستخدام طريقة دورة الألعاب الجماعية. ج. الطلب من الطلاب المشاركة في عملية التعلم باستخدام طريقة دورة الألعاب

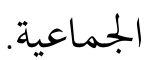

د. تسجيل الأحداث و الأشياء المهمة التي تحدث أثناء الدراسة. مرحلة الملاحظة أ. مراقبة و تسجيل عملية التعلم التي تحدث أثناء الدورة الثانية، وهذه الملاحظة قد أجرت الباحثة بمساعدة المراقب. ب. توثيق أنشطة التعليم و أنشطة الطلاب في الفصل الدراسي. مرحلة الانعكاس

أ. يناقش الباحث نتيجة الملاحظة مع المراقب للحصول على نتيجة الملاحظة

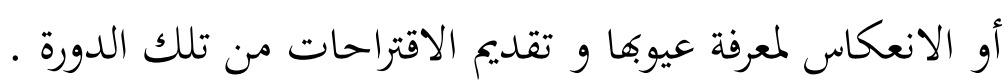
ب. نتيجة الانعكاس في الدورة الثانية.

النتيجة المطلوبة

النتيجة المطلوبة في هذا البحث هي ترقية نتيجة تعلم النحو باستخدام طريقة دورة الألعاب الجماعية. و سيقف هذا البحث إذا توفرت المعايير المعنية كالتالية: (1) عملية 
التعليم و التعلم مناسبة بخطة التعليم باستخدام طريقة دورة الألعاب الجماعية. r) تبلغ

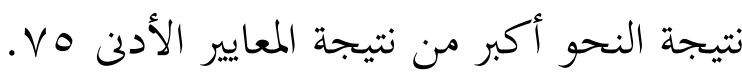

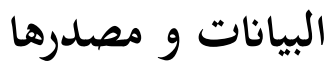

البيانات التي تم الحصول عليها في هذا البحث على البيانات الكمية والبيانات

النوعية. البيانات الكمية هي البيانات التي تحصل من نتيجة الاختبار في فهاية كل دورة و من نتيجة ورقة العمل للطلاب. و مصدر البيانات من هذا البحث هو الباحث والمراقب وطلاب الفصل العاشر وعددهم ·ـ طالبا. والبيانات النوعية هي البيانات التي تحصل من ملاحظة عملية التعلم ومن نتيجة الوثائق خلال عملية التعلم.

\section{مفهوم دورة الألعاب الجماعية}

إن طريقة دورة الألعاب الجماعية هي طريقة التعلم التعاوني تركز على أهمية المسابقة بين الطلاب، يتم تنفيذ المسابقة لتحسين قدرة أعضاء المجموعة في المسابقة

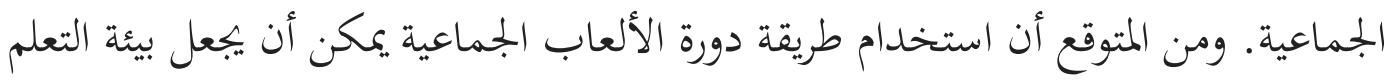
أكثر متعة (محمد محمود الحيلة، ه . . ب)، والطلاب يشتركون في عملية التعليم والتعلم بالجلد، وترقية أنشطة تعلم الطلاب ونتائج النحو.

تم تطوير دورة الألعاب الجماعية في الأصل بواسطة Devied Devries و مeith

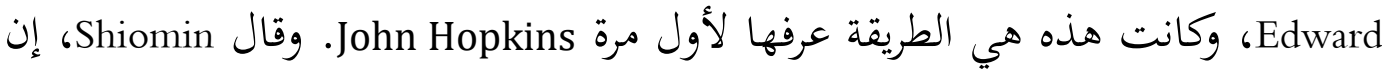
طريقة دورة العاب الجماعية هي طريقة تعلم تعاونية سهلة التنفيذ، تشمل أنشطة جميع الطلاب دون الحاجة إلى وجود اختلافات في الحالة، بما في ذلك دور الطلاب كمدرسين زملاء وتحتوي على عناصر من اللعب والتعزيز. و و قال سلافين، إن طريقة دورة العاب الجماعية هي دورة أكاديمية، ويستخدم الاختبارات وأنظمة درجات التقدم الفردية، حيث يتنافس الطلاب كممثلين لفريقهم مع أعضاء آخرين في الفريق يكون أدائهم الأكاديمي

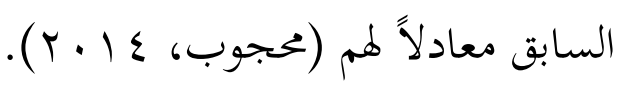

ورأى ويكاندري أن دورة ألعاب الجماعية قد استخدمت في مجموعة متنوعة من

الموضوعات، ومن الأفضل استخدامها لتدريس أهداف التعلم التي صيغت بحدة مع إجابة 
واحدة صحيحة، مثل الحساب والتطبيق الذي يتميز بالرياضيات والحقائق ومفاهيم العلوم (Asnawati, 2017).

\section{مكونات دورة الألعاب الجماعية}

إن التعليم التعاوين نوع دورة الألعاب الجماعية يتكون من خمس مراحل التالية

(Priansa, 2017:310). وهي:أولا، التعليم: تتكون هذه المرحلة من مرحلتين مهمتين، هما الافتتاح وهو في بداية التعلم، يقدم المعلم المواد المراد تعلمها، و ألقى المعلم هداف الدراسة، وشجع الطلاب بالتعلم. خلال هذا الفصل الدراسي، يتعين على المعلم إعداد أوراق العمل

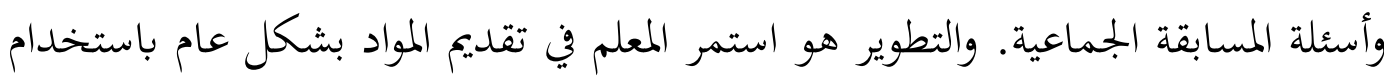
طريقة المحاضرة. في هذه المرحلة، طلب المعلم من الطلاب الانتباه وفهم المواد المقدمة. لأغها

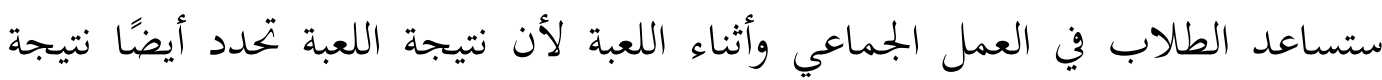
المجموعة.

ثانيا، الدراسة في المجموعات، يقرأ المعلم أعضاء المجموعة ويطلب من الطلاب التجمع وفقًا لمجموعاقم. تتكون المجموعات عمومًا من ع أو ه طلاب متنوعين. تتمثل وظيفة المجموعة في استكشاف المواد مع الأصدقاء بشكل أكثر تحديدًا لإعداد الأعضاء للعمل بشكل جيد ومثالي أثناء اللعبة. المجموعات هي الجزء الرئيسي من دورة الألعاب الجماعية. بعد إكمال التعلم الجماعي، يطلب المعلم من ممثلي المجموعة تقديم نتائج العمل الجماعي. في هذه الطريقة، يعمل المعلم كالميسر للالتفاف في بجموعات إذا كانت هناك مجموعات تواجه صعوبات.

ثالثا، لعبة (Games) تتكون اللعبة من أسئلة مصممة لاختبار معرفة الطلاب التي تم الحصول عليها من خلال الفصل الدراسي والتعلم الجماعي. بشكل عام، تتكون اللعبة من أسئلة بسيطة مرقمة، والتي تتم كتابتها على نفس الورقة. يختار الطلاب البطاقات المرقمة

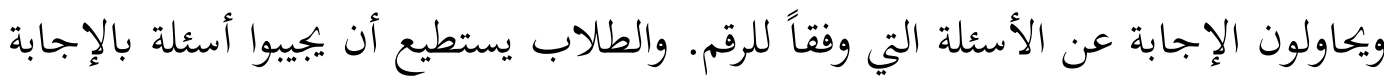
الصحيحة ويحصلون على النتيجة. 
رابعا، المسابقة الجماعية (Tournament) هي بنية اللعبة. تقام المسابقة الجماعية

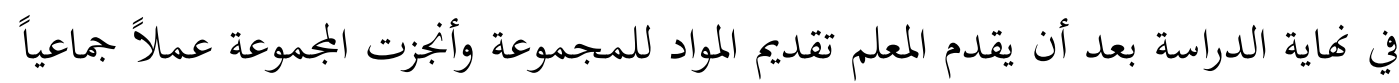

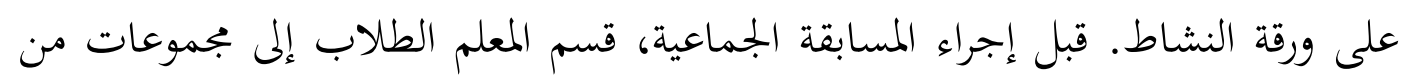

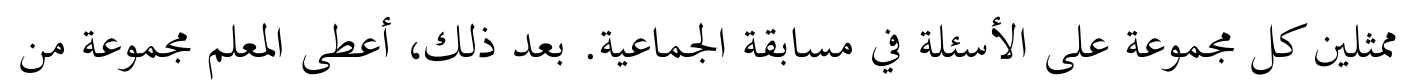

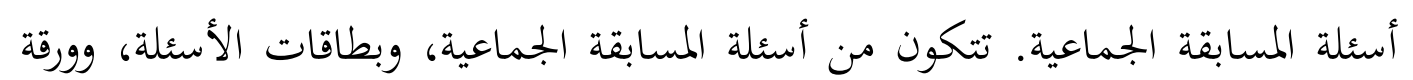

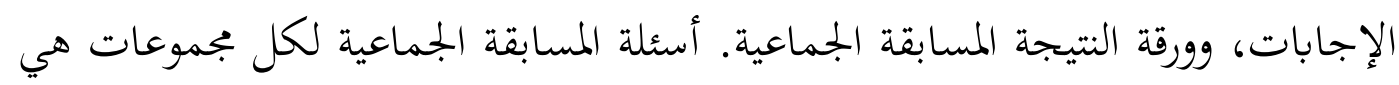
نفسها. خامسا، جوائز المجموعة (Team Recognize) في هذه المرحلة، تقديم الهدايا للمجموعة التي حصلت على أعلى الدرجات. تحصل كل مجموعة على شهادة وجائزة إذا

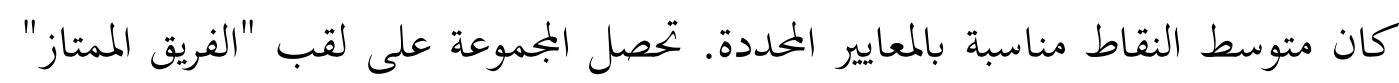

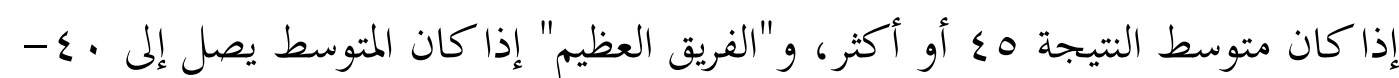

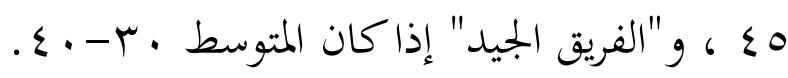

إن نتيجة التعلم هي القدرات التي لدى الطلاب بعد تلقي تجربة التعلم الخاصة بهم.

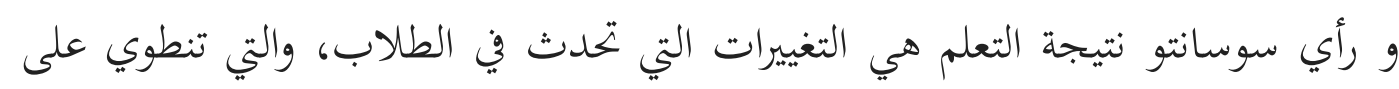

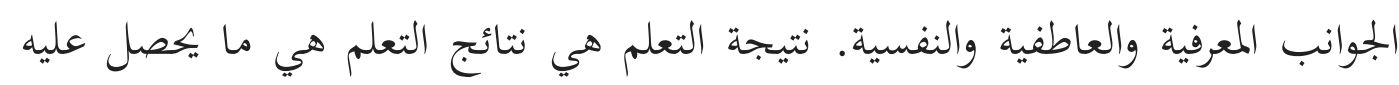

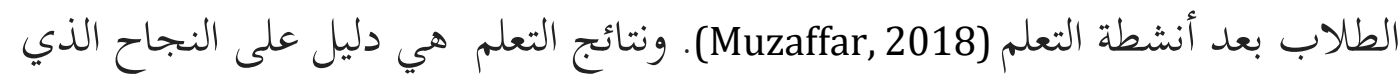
حققه الطلاب حيث يمكن أن يؤدي كل نشاط تعليمي إلى تغيير محدد. في هذه الحالة،

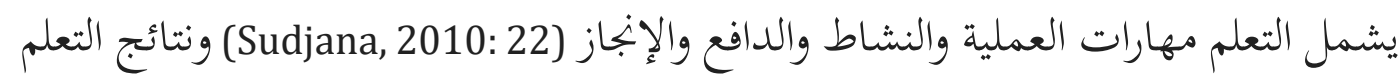

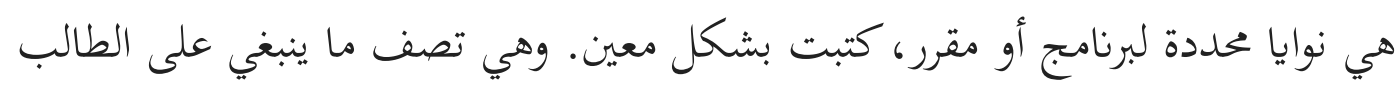

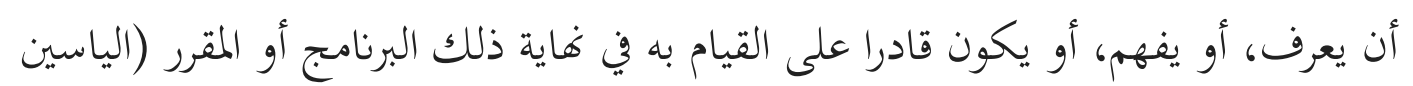

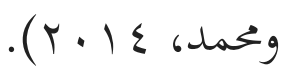

\section{دور القواعد النحوية في تعلم اللغة العربية}

كانت اللغة العربية لها دور مهم في مجال التربية والعلوم الإسلامية. واللغة العربية

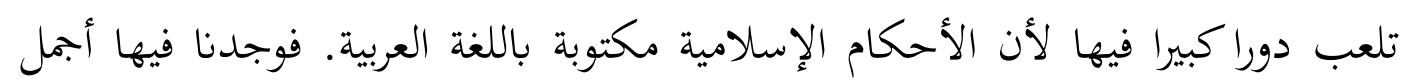


ترتيب الكلمات والجمل والأساليب. ليس تعلم اللغة العربية عملية سهلة ولها القواعد المتعددة التي يمكن أن يفهمها الطلبة جيدا. وأما دور القواعد النحوية في تعلم اللغة العربية فهو كما يلي: تساعد الطلاب في تحسين أشكال كل كلمات عربية، وتساعد الطلبة في فهم عناصر الجمل في النص، وتساعد الطلبة في إتيان الأمثلة الجلديدة، وتساعد الطلبة في

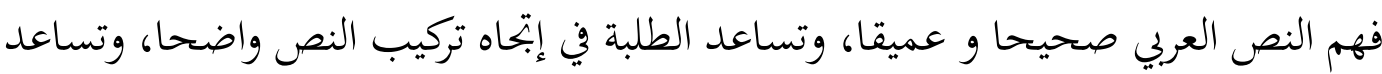
الطلبة في دقة التعبير وسلامة الأداء وضبط الكلمات \&arzun, Husnizar, \& Halimahtussakdiah, 201). إن هدف القواعد النحوية لا يخالف ههدف اللغة الأجنبية الأخرى. وهو تسهيل على فهم النصوص العربية أو الكلام العربي حتى وصول المعلومات إلى القارئ أو السامع.

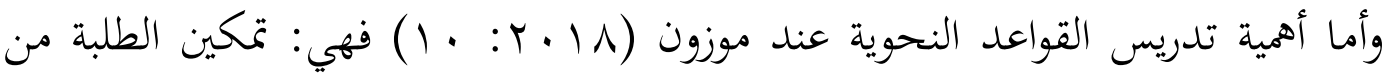
محاكة الأساليب الصحيحة لغويا، وجعل هذه المحاكاة مبنية على أساس مفهوم بدلا من

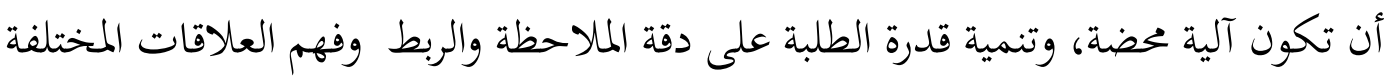

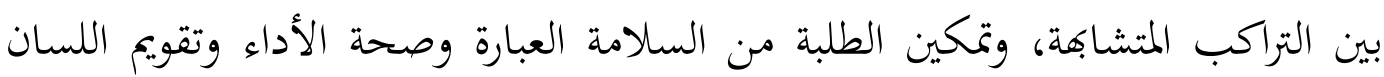
وعصمته من الخطأ في الكلام، تمكين الطلبة من الترتيب المعلومات وتنظيمها في أذهاهم و يدريبهم على دقة التفكير والعليل والاستنباط، ووقوف الطلبة على أوضاع اللغة وصيغها لأن قواعد النحو إنما هي وصف علمي دئ لتلك الاوضاع والصيغ وبيان للتغيرات التي تحدث في ألفاظها و فهم للاساليب المتنوعة التي يسير عليها اهلها.

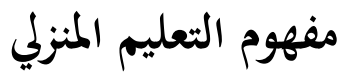

التعليم المنزلي أو ما يعرف بالمدرسة المنزلية هو الأنشطة التعليمية المستمرة والمنظمة

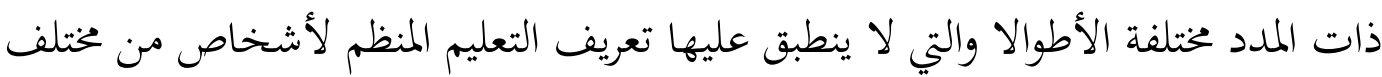
الأعمارا ومن الممكن أن يتم تنفيذ هذا التعليم داخل الموسسات التعليمية أو خارج نفوذها

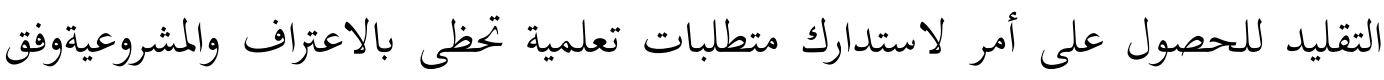

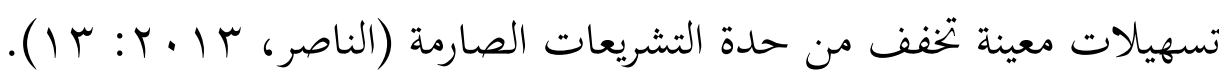


التعليم المنزلي هو نموذج لأنشطة التعليم أو التعلم التي يتم تنفيذها في المنزل أو في أماكن أخرى غير المدارس التقليدية سواء الزملاء (الأسرة) والمجتمع حيث التنظيم الذاتي لتنفيذ التعليم، والأهداف التعليمية، والقيم التي يجب أن تكون المتقدمة والذكاء والمهارات والمناهج والمواد، وكذلك أساليب التعلم والممارسات. المصطلحات الأخرى التي تشير إلى ولى ولى التعليم المنزلي هي التعليم المنزلي، التعلم في المنزل أو المدارس المستقلة. يتم التعليم المنزلي من

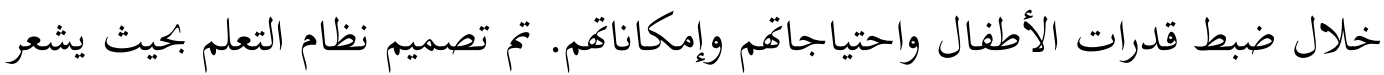
الأطفال بالسعادة والراحة، ولا يشعرون بالإرهاق ولا يشعرون بالعبء في التعلم لتحقيق نتائج تعليمية مثالية (Sumardiono, 2014:6).

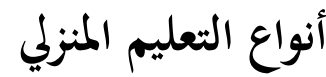

هناك ثلاثة أنواع من التعليم المنزلي عند موليادي وهي: (1) التعليم المنزلي الفردي، وهذا

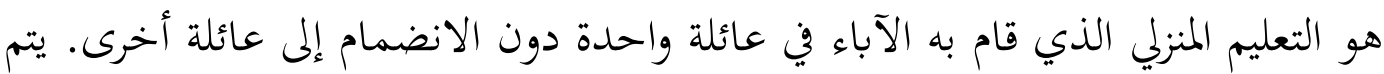
تنفيذ التعليم المنزلي من هذا النوع من أجل أغراض أو أسباب محددة لا يمكن أن تكون

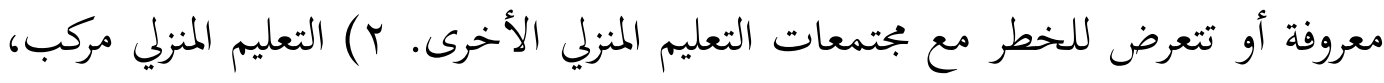

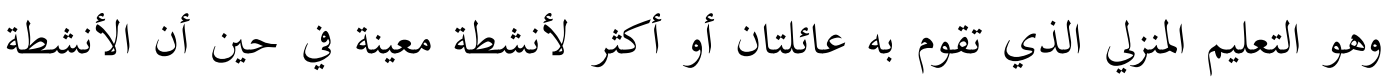

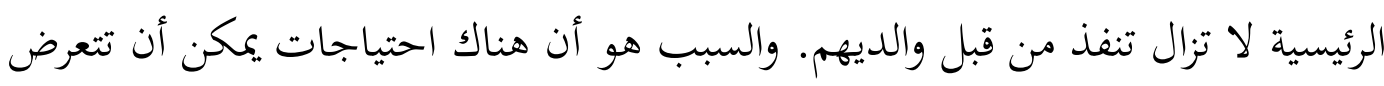
للخطر بعض الأسر للقيام بأنشطة مشتركة. r) بجتمع التعليم المنتلي، هو مزيج من العديد من التعليم المنزلي المركب الذي يجمع ويحدد المنهج والمواد التعليمية والمرافق والبنية التحتية وكذلك جداول التعلم.

\section{نتيجة إجراء العمل في الدورتين}

أجري هذا البحث في الدورتين، الدورة الأولى مبدوءة في با ا من مارس والدورة

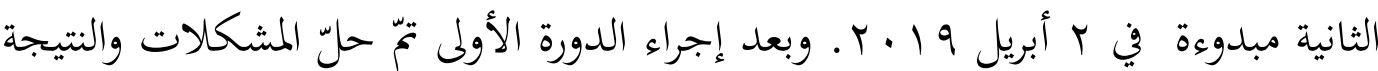
هي: 1) أكثر الطلاب لم يفهمو طريقة دورة الألعاب الجماعية، حتى تمنعهم عملية الألعاب إباء

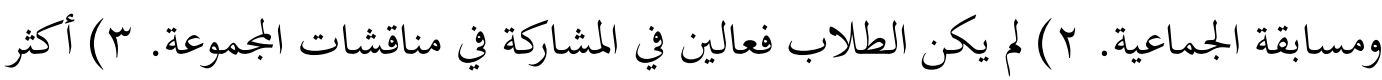


الطلاب لم يفهمو مادة النحو خاصةً عن الجملة الاسمية. ع) لم تتحقق نتائج تعلم الطلاب الأهداف المرجوة من البحث. والطلاب يحصلون على النتائج أصغر من معايير النجاح

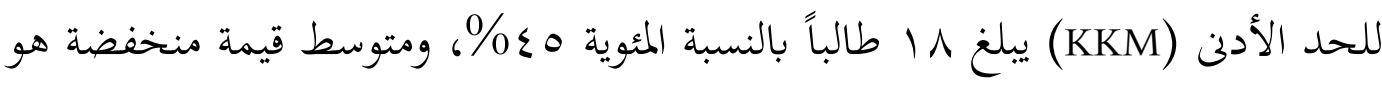
.$\vee \cdot 6 \wedge \vee$

وبعد إجراء الدورة الثانية قد حصل البحث على نتيجة مرجوة وهي كما يلي:

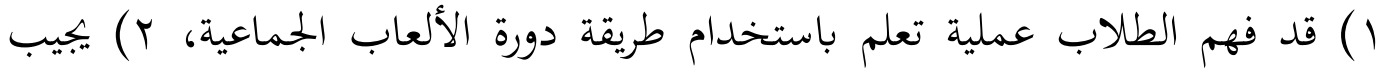
الطلاب الأسئلة في مرحلة لعبة بالإجابة الصحيحة. ب) يشرح الطلاب نتائج المناقشة في المجموعة دون خوف. ه) حدث تفاعل بين الطلاب وبين الطلاب والباحث في عملية الدراسة. 7) ومن نتائج الاختبار في الدورة الثانية حصلت على نتيجة مرجوة. و نتائج الطلاب أصغر من نتائج الكاملة للحد الأدنى (V0) هي ج ب طلاب، أو بالنسبة المئوية

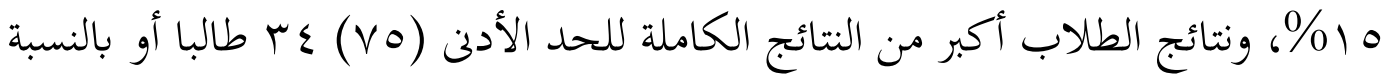

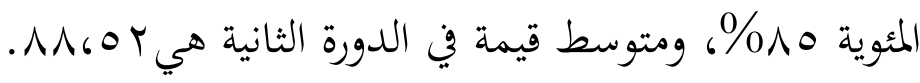

وصف نتائج تعلم النحو باستخدام طريقة دورة الألعاب الجمماعية في المدرسة المنزلية شعائر الله بيكاسي اعتمادا على الاختبار في فاية كل دورة، وجد الباحث البيانات التالية: الجدول ا. ـنتائج التعلم في الدورة الأولى

\begin{tabular}{|c|c|c|c|c|}
\hline $\begin{array}{c}\text { النسبة التراكمية } \\
\text { (\%) }\end{array}$ & $\begin{array}{c}\text { النسبة المئوية } \\
\text { (\%) }\end{array}$ & عدد الطلاب & جمموعة القيمة & الرقم \\
\hline 1. & 1. & $\varepsilon$ & $00-0$. & 1 \\
\hline ro & 10 & 7 & $71-07$ & r \\
\hline$r$. & 0 & r & $T V-T r$ & r \\
\hline 00 & ro & 1. & VT- $-1 \wedge$ & $\varepsilon$ \\
\hline V V6o & Tr60 & 9 & $v q-v \varepsilon$ & 0 \\
\hline
\end{tabular}




\begin{tabular}{|c|c|c|c|}
\hline $1 \ldots$ & Tr6o & 9 & $\Lambda_{0}-1$. \\
\hline & $\% 1 \ldots$ & $\varepsilon$. & الجموع \\
\hline
\end{tabular}

اعتمادا على الجدول السابق، إن أكثر الطلاب قد حصلوا على النتائج أصغر

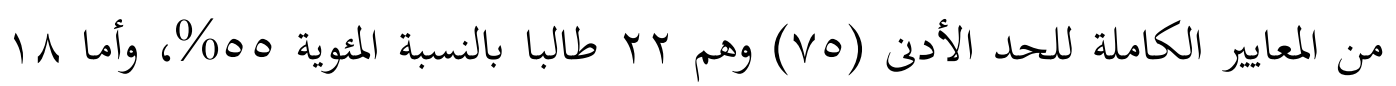

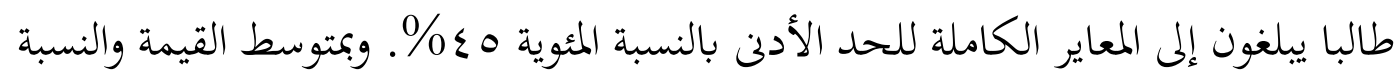
الكاملة الذي تم بيانه فتعتمد نتائج تعلم النحو باستخدام طريقة دورة الألعاب الجماعية في الفصل العاشر في المدرسة المنزلية بيكاسي لم تصل إلى المعايير الكاملة للحد الأدنى

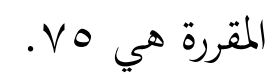

والنتائج المحصولة في الدورة الأولى مرسومة في هستوكرام وفولغون التاليتان:

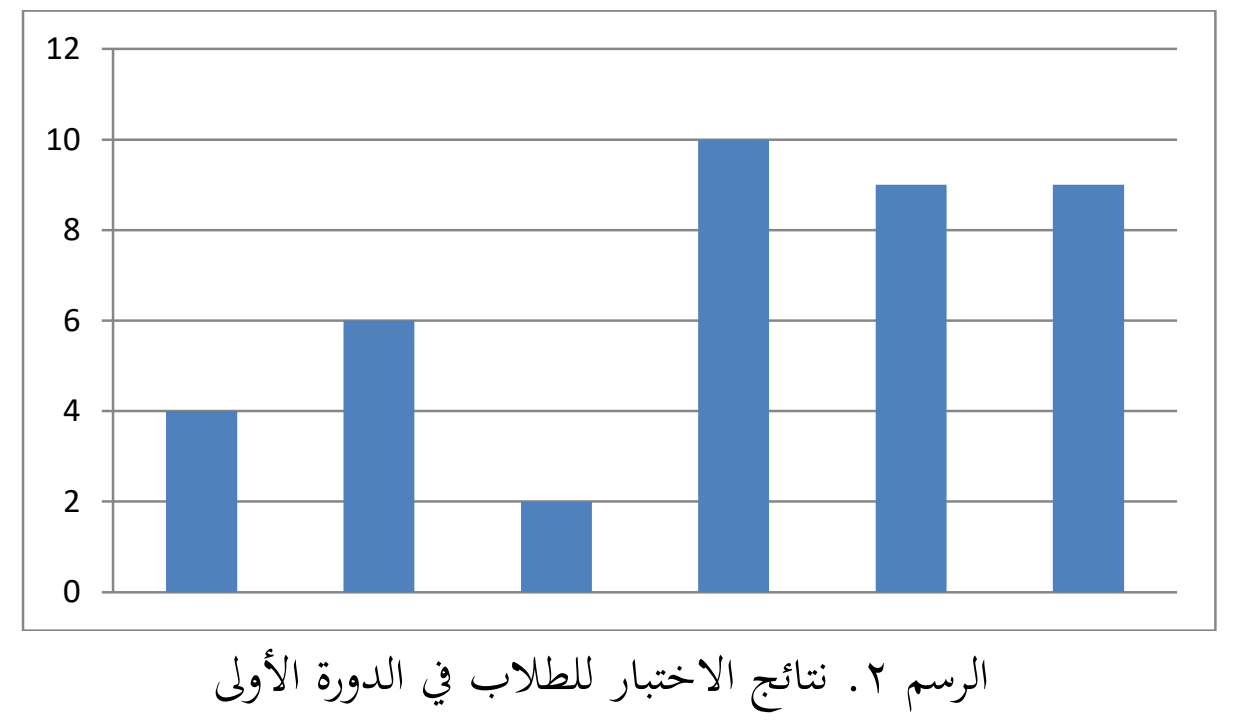

الجدول r ـ نتائج التعلم في الدورة الثانية

\begin{tabular}{|c|c|c|c|c|}
\hline التراكمية (\%) النسبة & $\begin{array}{c}\text { النسبة المئوية } \\
\text { (\%) }\end{array}$ & الطلاب & جموعة القيمة & الرقم \\
\hline 10 & 10 & 7 & $V T-V Y$ & 1 \\
\hline ro & 1. & $\varepsilon$ & $\Lambda I-V V$ & r \\
\hline$\varepsilon 0$ & $r$. & $\Lambda$ & $\Lambda T-\wedge r$ & r \\
\hline
\end{tabular}




\begin{tabular}{|c|c|c|c|c|}
\hline$V V$ & $1 \cdot$ & $\varepsilon$ & $91-\Lambda V$ & $\varepsilon$ \\
\hline Vo & $r \cdot$ & $\wedge$ & $97-q r$ & 0 \\
\hline \multirow[t]{2}{*}{$1 \cdots$} & ro & 1. & $1.1-9 V$ & 7 \\
\hline & $\% 1 \ldots$ & $\varepsilon$ & & \\
\hline
\end{tabular}

اعتمادا على الجدول السابق، أن أكثر الطلاب قد حصلوا على نتائج أصغر

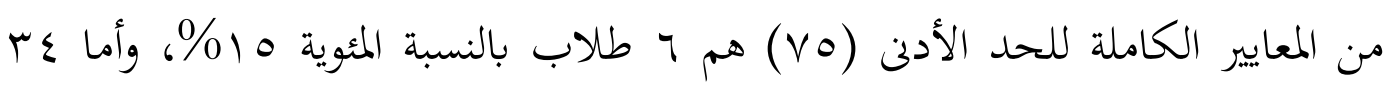

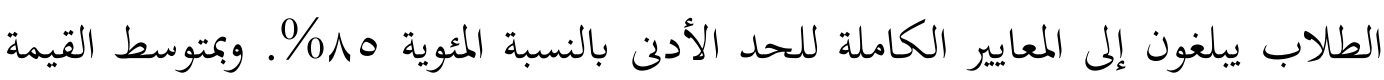
والنسبة الكاملة الذي تم بيانه فتعتمد نتائج تعلم النحو باستخدام طريقة دورة الألعاب

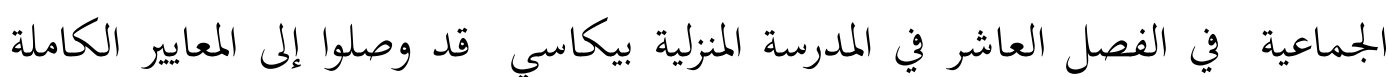

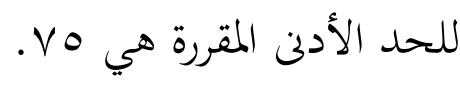

والنتائج المصصولة في الدورة الثانية مرسومة في هستوكرام وفولغون التاليتان:

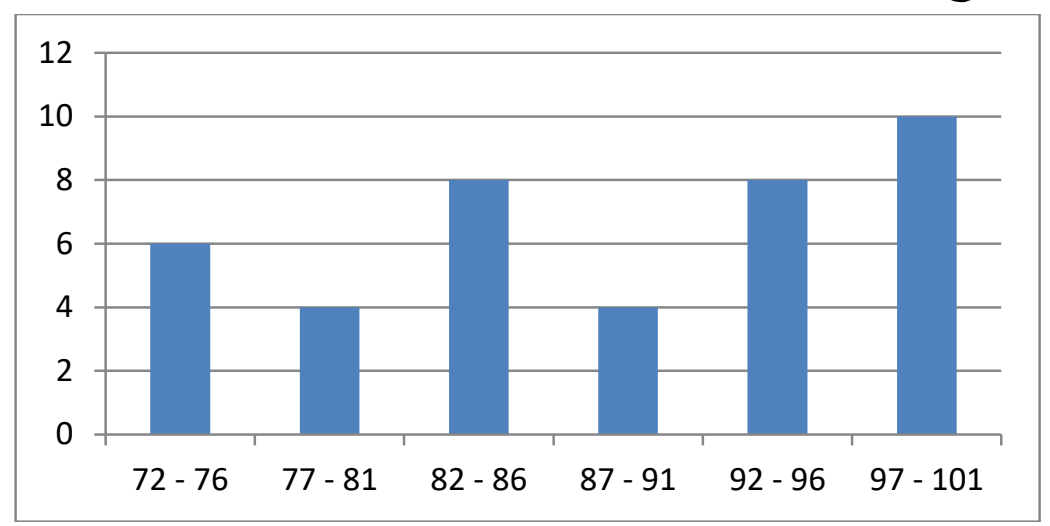

الرسم r. نتائج الاختبار للطلاب في الدورة الثانية

الجدول بـ مقارنة نتائج البحث بين الدورة الأولى والدورة الثانية

الترقية

متوسطة قيمة نتيجة

متوسطة قيمة نتيجة

الاختبار في الدورة الأولى الاختبار في الدورة الثانية

18670

NN6OY

$V \cdot 6 \wedge V$ 
اعتمادا على جدول السابق، أن متوسط قيمة نتائج الاختبار في مادة النحو

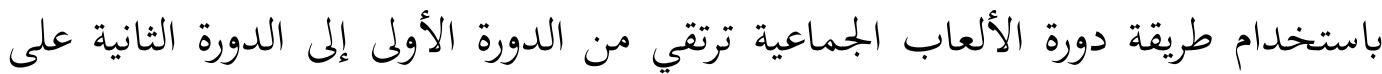

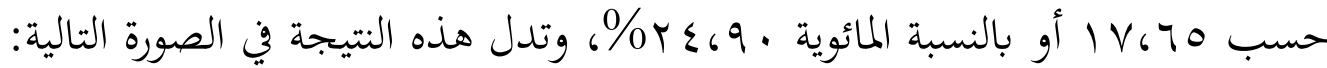

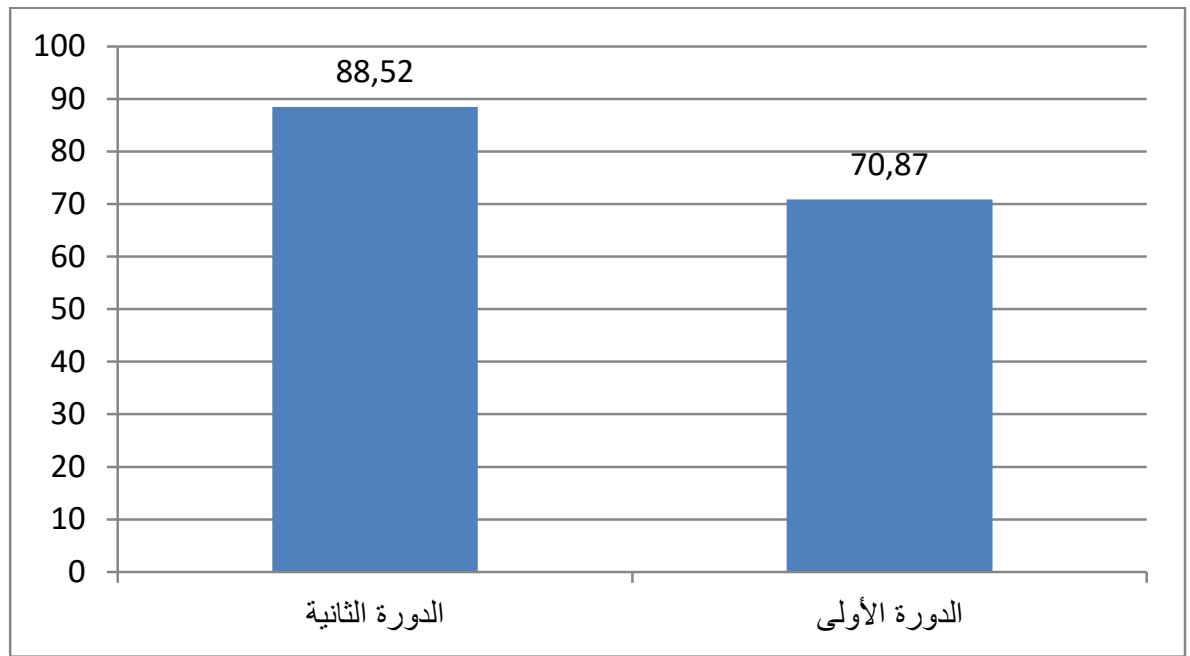

الرسم ع ـ مقارنة متوسطة القيمة في الدورة الأولى والدورة الثانية

وأما شرح مقارنة النسبة الكاملة للطلاب كالتالي:

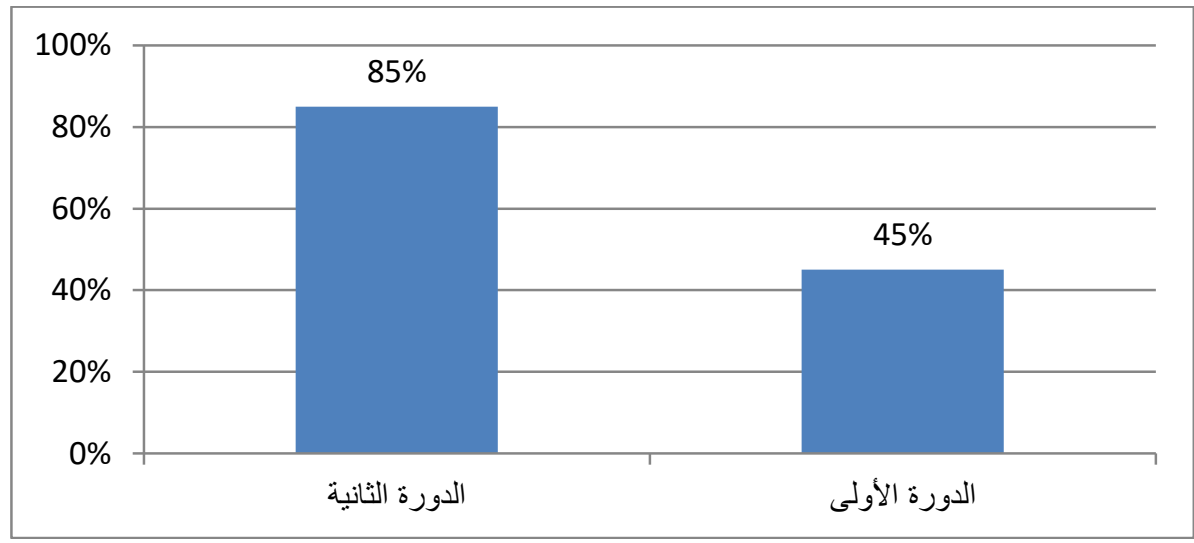

الرسم ه. النسبة الكاملة للطلاب في الدورة الأولى والدورة الثانية

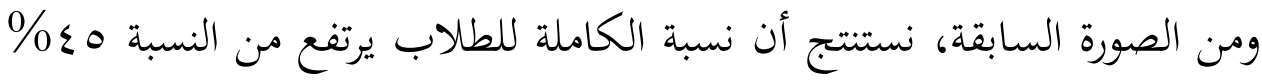
إلى 01\% ويمكن استنباط هذه الشرح هناك ترقية نتيجة تعلم النحو باستخدام طريقة دورة

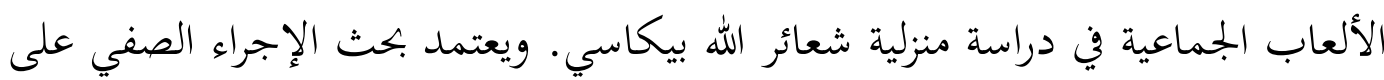
ترقية نتيجة تعلم النحو لدى الطلاب باستخدام طريقة دورة الألعاب الجماعية في الفصل 
العاشر في المدرسة المنزلية بيكاسي. وتظهر نتائج البحث الأخيرة بعد إجراء البحث في

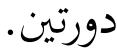

حصل الباحث على نتيجة البحث من مصادر البحث والبيانات التي تمّ الحصول عليها أثناء العملية الدراسية، وأخيرا بالنظر إلى نتائج الاختبار ـ ومن ملاحظة البحة البيانات في نتائج الاختبار، توجد ترقيةنتائج تعلم النحو بشكل واضح. وبعد ملاحظة عملية التعليم والتعلم، يترقّى نشط الطلاب في تعلم اللغة العربية. وهناك التفاعل بين الطلاب، وبين

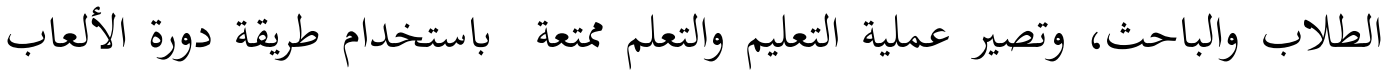
الجماعية.

الحخلاصة

إن للمدرس دور هام في عملية التعليم لتحديد التخطيط وجودة التعليم. فلذلك لابد للمدرس أن يحاول بإعداد واستخدام طريقة التعليم والوسيلة التعليمية المناسبة بحاجة الطلاب. وعلى المدرس أن يخطط عملية التعليم بأحسن الخطوات، ثم يقوم المدرس بانعكاس العملية التعليمية السابقة ثم إصلاحها لترقية وتحسين عملية التعليم في القادمة. واستخدام المدرس طريقة دورة الألعاب الجماعية كطريقة التعلم التعاوني في تعليم اللغة العربية خاصة علم النحو لطلاب الفصل العاشر في المدرسة المنزلية شعائر الله بيكاسي

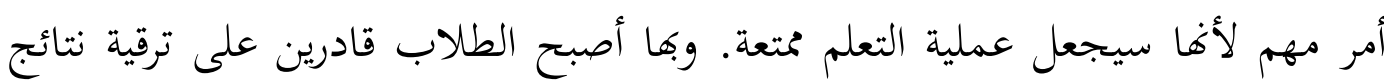
تعلم النحو، وتزوّد حماستهم في التعلم. ويجعل الطلاب ناشطين في طرح الأسئلة والإجابة. كما أفم متحمسون لممارسة استخدام الطريقة التعليمية بطريقة دورة الألعاب الجماعية.

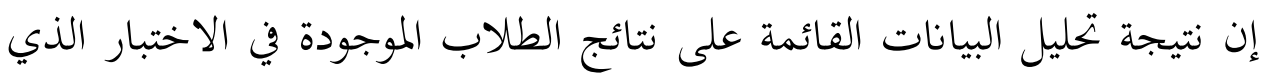
تم إجراؤه، يبدوا أن الطلاب الذين حصلوا على النتائج أصغر من المعايير من الكاملة للحد

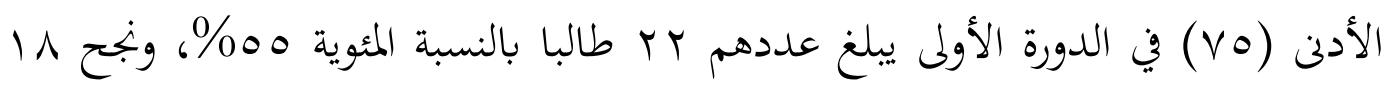

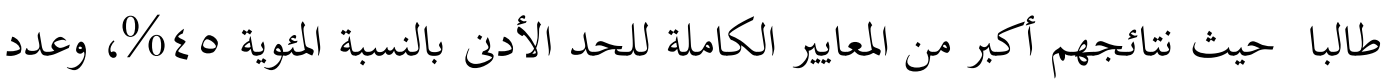

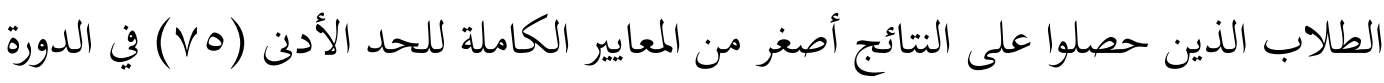

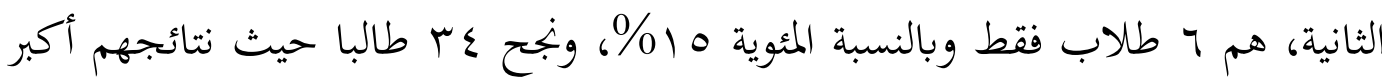


من المعايير الكاملة للحد الأدنى بالنسبة المئوية هم\%\%. وبملاحظة النتائج الدراسية في هذا البحث، توجد ترقية نتائج تعلم النحو باستخدام طريقة دورة الألعاب الجماعية لدى طلاب

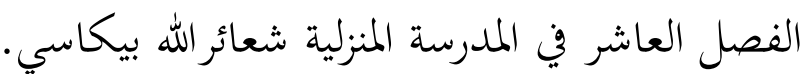

محمد محمود الحيلة. أثر التعُّم التعاوني القائم على مجموعات الخبراء في التحصيل المباشر. .$r .0$

إبنية، خ. ع. فاعلية /ستراتيجية مقترحة قائمة على التعلم التعاوني في تنمية مهارات التعبير الكتابي لدى طلبة الصف التاسع الأساسي في ملديرية التربية والتعليم لمنطقة القصر.

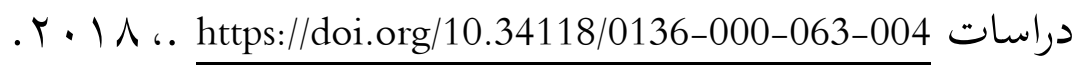

الناصر، ع. ا. س. التعليم غير النظامي في الأردن. عمان: وزارة التربية والتعليم الأردن، .$r \cdot 1 r$

الياسين، و. س. ومحمد، ا. م. ي. استراتيجية التعلم التعاوني وعلاقتها بناعلية الإدارة

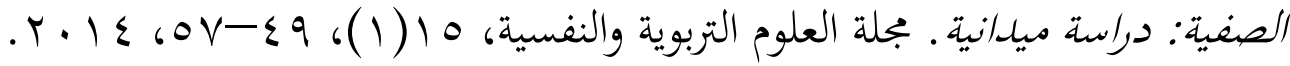

IZDIHAR. توفيق، أ. دافعية استخلام التعلم التعاوني لتنمية تعليم مهارة الكلام .r. $\mid \leq$ https://doi.org/10.22219/izdihar.v1i1.6564

محجوب, م. ص. التعلم التعاوني: أسسه واستراتيجياته وتوجيهات لتطبيقه في تعلم العربية

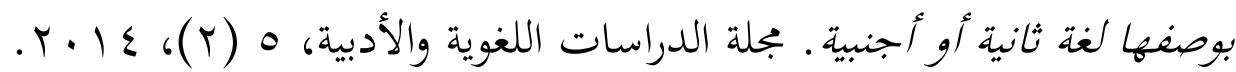

Anugrah, M. Penelitian Tindakan Kelas. Yogyakarta: Leutika Nouvalitera, 2019.

Asnawati, S. Peningkatan Kemampuan Komunikasi Matematis Siswa Smp Dengan Pembelajaran Kooperatif Tipe Teams-Gamestournaments. Euclid. https://doi.org/10.33603/e.v3i2.332, 2017.

Marzun, R., Husnizar, H., \& Halimahtussakdiah, H. Ta'lîm al-qawâ'id annahwiyyah bima'had syams ad-dhuha. LISANUNA: Jurnal Ilmu Bahasa Arab dan Pembelajarannya, 8 (2), 133, 2019. 
Muzaffar, A. Derivasi Indikator Hasil Belajar Bahasa Arab. LISANUNA: Jurnal Ilmu Bahasa Arab dan Pembelajarannya. https://doi.org/10.22373/l.v7i2.3465, 2018.

Priansa, D. J. Pengembangan Strategi dan Model Pembelajaran. Bandung: Pustaka Setia, 2017.

Sudjana, N. Dasar-Dasar Proses Belajar Mengajar. Bandung: Sinar Baru Algensindo, 2010.

Sumardiono. Apa Itu Homeschooling. Jakarta: Panda Media, 2014.

Wasilah, W., \& Nurlina, N. Tathbîq Namûdzaj Al-Ta'allum Al-Ta'âwuni Bi Nau' Tarqîm Al-Ro's Li Tarqiyyah Mahârah Al-Qirâah Li Al-Tullâb Fî Al-Fashl Al-Sâbi' Bi Al-Madrasah Al-Tsanâwiyyah Aisyah Palembang. Taqdir. https://doi.org/10.19109/taqdir.v4i1.2163, 2018.

Wibowo, N. Pembelajaran Berdasarkan Gaya Belajar Di Smk Negeri 1 Saptosari. Jurnal Electronics, Informatics, and Vocational Education (ELINVO). https://doi.org/10.31436/ills.v5i2.168, 2014 